\title{
STUDY OF THE MAGNETIC COUPLING BETWEEN QUADRUPOLE AND DIPOLE CORRECTOR MAGNETS FOR THE KEK B-FACTORY
}

\author{
K.Egawa, M.Masuzawa, KEK, Tsukuba, Japan
}

\begin{abstract}
Most of the quadrupole magnets in the KEK B-factory are accompanied by a set of vertical and horizontal dipole corrector magnets in both the LER and HER rings[1]. The typical separation between a quadrupole and a dipole corrector magnet is $202 \mathrm{~mm}$, as measured from the laminated ends. This corresponds to $\sim 3.7$ (2.4) times of the bore radius of a quadrupole magnet in the arc (straight) sections. Since the series measurements of quadrupole magnets were performed without any magnetic material nearby[2], the effect of the magnetic coupling from the corrector dipole magnet in the actual beam line needs to be measured. Measurements of the coupling between quadrupole and dipole corrector magnets are presented.
\end{abstract}

\section{INTRODUCTION}

Magnetic coupling between a quadrupole and a dipole corrector magnet is measured with two systems, a harmonic coil system and a mapping system[3]. The harmonic coil system is used to measure each of the field components with and without a magnet nearby at various separations between the two magnets. The mapping system is used to measure the field distribution along the beam line to obtain the effective length. Table 1 shows the parameters of the magnets discussed in the following sections.

Table 1: LER Magnet parameters

\begin{tabular}{|l|c|c|c|}
\hline $\begin{array}{c}\text { Magnet } \\
\text { name }\end{array}$ & Magnet type & $\begin{array}{c}\text { Lamination } \\
\text { length }(\mathrm{mm})\end{array}$ & $\begin{array}{c}\mathrm{r}(\mathrm{mm}) \\
\text { or } \mathrm{g} / 2\end{array}$ \\
\hline QA & quadrupole & 400 & 55 \\
\hline Qrf & quadrupole & 500 & 83 \\
\hline $\mathrm{StV}(\operatorname{arc})$ & dipole corrector & 200 & 76.5 \\
\hline $\mathrm{StH}(\operatorname{arc})$ & dipole corrector & 200 & 58 \\
\hline $\mathrm{StV}($ wide $)$ & dipole corrector & 200 & 100 \\
\hline StH(wide) & dipole corrector & 200 & 86 \\
\hline
\end{tabular}

Note: QA and Qrf represent quadrupole magnets in the LER arc sections and straight(RF) sections, respectively. $\mathrm{StV}(\operatorname{arc})$ and $\mathrm{StH}(\operatorname{arc})$ are the vertical and horizontal dipole corrector magnets in the arc sections. $\mathrm{StV}$ (wide) and $\mathrm{StH}$ (wide) are the vertical and horizontal dipole corrector magnets for the straight sections, respectively.

\section{EFFECT ON THE QUADRUPOLE FIELD}

\subsection{Effect on the main quadrupole field}

A $2.5 \mathrm{~m}$ harmonic coil is used to measure the magnetic coupling between the quadrupole and dipole corrector magnets as shown in Fig. 1. The quadrupole field is measured without a dipole corrector magnet first. A dipole corrector magnet is placed at various distances and the field of the quadrupole magnet is measured at each distance. No current is applied to the dipole corrector magnet during the measurement.

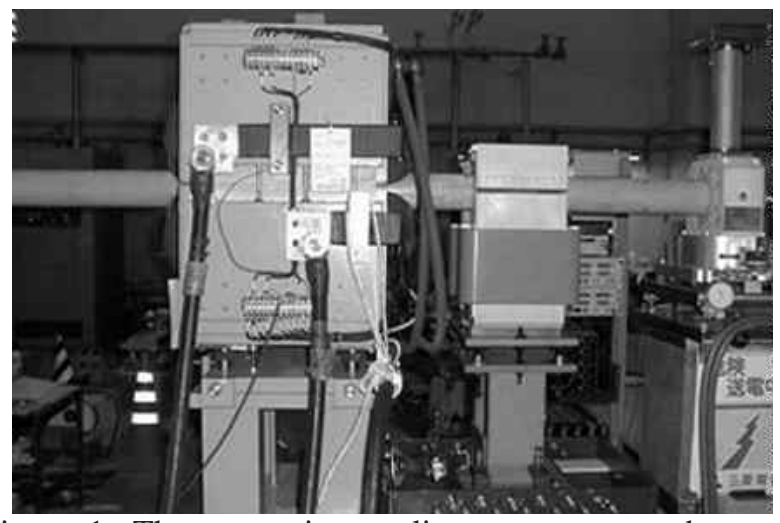

Figure 1: The magnetic coupling measurement between QA (on the left) and $\mathrm{StV}(\operatorname{arc})$ by a harmonic coil.

Fig. 2 shows the magnetic coupling effect on the QA integrated quadrupole field when there is an $\mathrm{StV}(\operatorname{arc})$ or an $\mathrm{StH}(\operatorname{arc})$ nearby. The field is normalized to the case where there is no dipole corrector magnet nearby and plotted against the separation. At a separation of $202 \mathrm{~mm}$ (the standard position in the KEKB rings) the integrated main quadrupole field $\left(\mathrm{B}^{\prime} \mathrm{L}\right)$ is reduced by $0.1 \%$ and $0.09 \%$ for the combinations of [QA, $\mathrm{StH}(\operatorname{arc})]$ and [QA, $\operatorname{StV}(\operatorname{arc})]$, respectively. The other combination, [Qrf, $\mathrm{StV}$ (wide)] shows a larger coupling effect of $0.2 \%$ at the same separation. This is due to the larger Qrf bore radius.

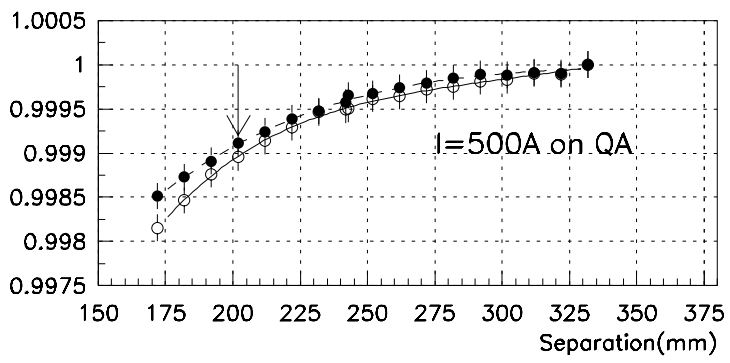

Figure 2: B'L/(B'L with no dipole corrector magnet nearby) is plotted as a function of the separation. The open and solid circles correspond to the combinations of [QA, StH(arc)] and [QA, StV(arc)], respectively. The arrow indicates the standard position of $\mathrm{StV}(\operatorname{arc})$ and $\mathrm{StH}(\operatorname{arc})$ with respect to QA in the KEKB tunnel.

The magnetic coupling dependence on the QA field strength (i.e., QA current) is measured. Figs. 3(a) and (b) 
show the coupling effect as a function of QA current. As seen, the current dependence is not significant.

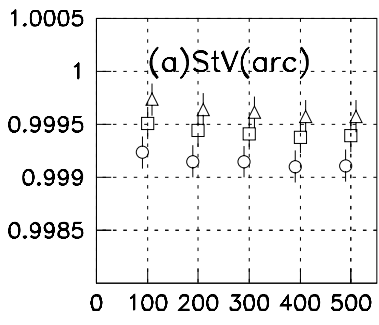

I(A)

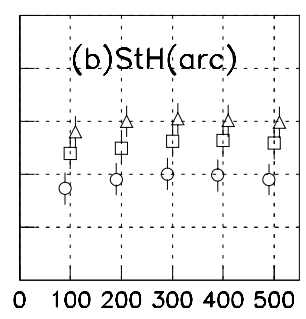

I(A)
Figure 3: Magnetic Coupling dependence plotted against various QA currents $(100 \mathrm{~A}, 200 \mathrm{~A}, 300 \mathrm{~A}, 400 \mathrm{~A}$ and $500 \mathrm{~A})$ for the combinations of [QA, $\mathrm{StV}(\operatorname{arc})]$ (a) and [QA, $\mathrm{StH}(\operatorname{arc})](\mathrm{b})$. The open circles, squares and triangles correspond to a separation of $202 \mathrm{~mm}, 222 \mathrm{~mm}$ and $242 \mathrm{~mm}$, respectively.

\subsection{Effect on the higher order multipole components}

In Fig. 4, the effect on the multipole components is shown for the [Qrf, StV(wide)] combination. As mentioned, this combination shows a larger coupling effect than the [QA, StV(arc)] combination. However, no significant effect from the magnetic coupling is observed in the higher order multipole components.

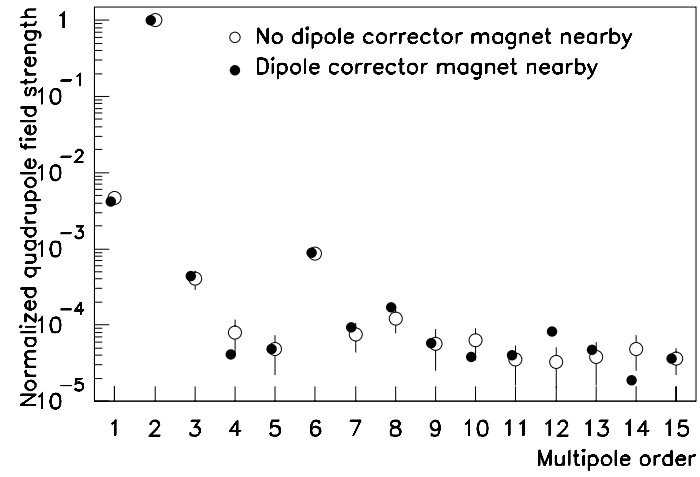

Figure 4: Normalized multipole components of Qrf are plotted. Open circles show the case when there is no dipole corrector nearby. Error bars indicate one standard deviation. Solid circles correspond to the case when a $\mathrm{StV}$ (wide) is placed at its standard location.

\subsection{Effect on the effective length}

Fig. 5 shows the mapping system used to obtain the field distribution along the beam line. The effective length, $\mathrm{L}_{\text {eff }}$, of QA is calculated from the field distribution shown in Fig. 6(a). $\mathrm{L}_{\text {eff }}$ changes from $458.99 \mathrm{~mm}$ to 458.60 if $\mathrm{StV}(\operatorname{arc})$ is placed at the standard position. The reduction in $\mathrm{L}_{\text {eff }}$ is $0.085 \%$, which agrees with the $\Delta \mathrm{B}^{\prime} \mathrm{L}$ measurement made with the harmonic coil discussed in section 2.1. The right side of the field distribution is distorted as shown in Fig. 6(b), which indicates that the field is partially absorbed by $\mathrm{StV}(\operatorname{arc})$. Fig. 6(c) shows the difference between the distorted and the original distributions. The absorbed field peaks at $\mathrm{z}=202 \mathrm{~mm}$, where the $\mathrm{StV}(\operatorname{arc})$ lamination begins. The normalized absorbed field is plotted in Fig. 6(d). Almost 100\% of the quadrupole field is absorbed by $\operatorname{StV}(\operatorname{arc})$ at $\mathrm{z}=300 \mathrm{~mm}$, which corresponds to the center of the dipole corrector magnet. Table 2 summarizes the coupling effects on QA.

Table 2: Magnetic coupling effect on QA

\begin{tabular}{|l|c|c|}
\hline \multicolumn{1}{|c|}{ corrector dipole } & $\Delta \mathrm{B}^{\prime} \mathrm{L}$ & $\Delta$ center $(\mathrm{mm})$ \\
\hline $\mathrm{StV}(\operatorname{arc})$ & $0.09 \%$ & 0.236 \\
\hline $\mathrm{StH}(\operatorname{arc})$ & $0.10 \%$ & 0.275 \\
\hline
\end{tabular}

Note : The magnetic center moves away from the nearby dipole corrector magnet.

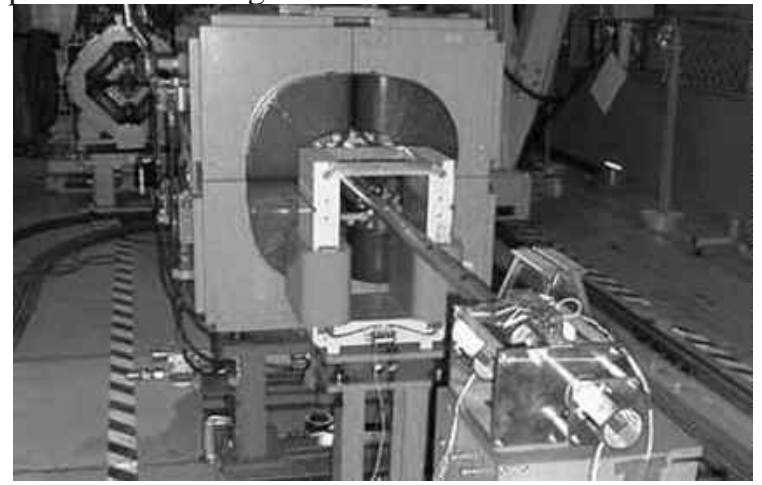

Figure : 5 Mapping system

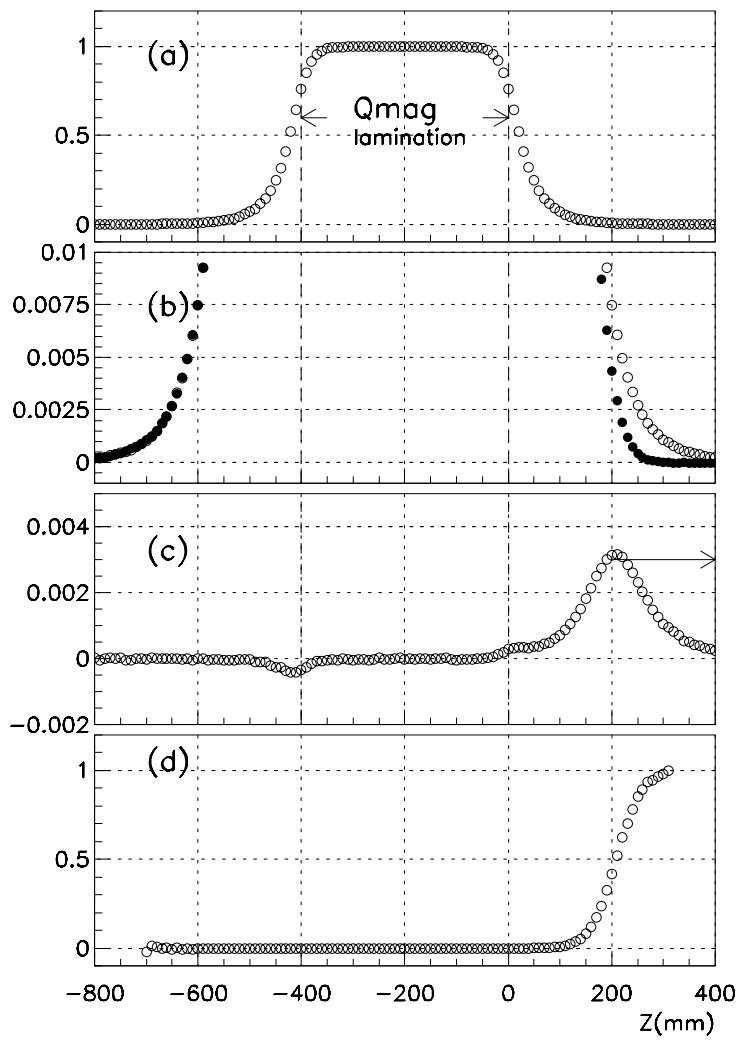

Figure 6: (a) The field distribution along the beam line. $\mathrm{z}=0$ corresponds to the position where QA lamination begins. (b) Two cases with $\mathrm{StV}(\operatorname{arc})$ (solid circle) and without StV(arc) (open circle). (c)Difference between the two distributions in (b). Difference distribution (c) is normalized by distribution (a) and plotted in (d). 


\section{EFFECT ON THE DIPOLE CORRECTOR FIELD}

The dipole corrector field also becomes smaller due to the presence of a quadrupole magnet nearby. Fig. 7(a) shows the field distribution of $\mathrm{StV}$ (wide) along the beam line with and without a quadrupole magnet in the standard position. A distortion of the field is observed near the quadrupole magnet. The difference between the two distributions is plotted in Fig. 7(b). $\mathrm{L}_{\text {eff }}$ is reduced from $390.27 \mathrm{~mm}$ to $358.29 \mathrm{~mm}$, which corresponds to a $8.9 \%$ decrease in BL (kick angle). The dipole magnetic field is absorbed mainly at $\mathrm{z}=300 \mathrm{~mm}$, where the quadrupole lamination begins, as is shown Fig. 7(c). The effect on the integrated dipole field is also measured by the harmonic coil system. The field reduction is measured to be $8.5 \%$. This indicates that the magnetic coupling effect takes place locally and does not effect the field in the center of the magnet.

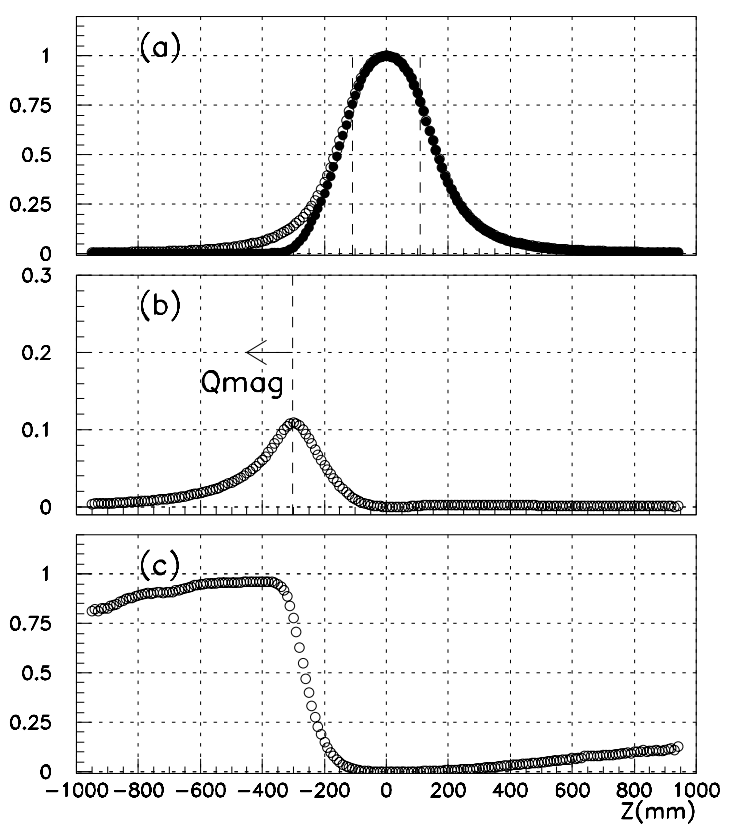

Figure 7: (a) $\mathrm{StV}$ (wide) field distribution along the beam line with (solid circle) and without (open circle) a quadrupole magnet. The field is distorted by the presence of a quadrupole magnet. (b) The difference between the two distributions in (a). (c)Absorbed field normalized by the original field.

The partial integrated dipole field strength, i.e., $\Sigma \mathrm{B}(\mathrm{z})(\mathrm{sum}$ from $\mathrm{z}=-950 \mathrm{~mm}$ to $\mathrm{z}$ ), is plotted as a function of $\mathrm{z}$ in Fig. 8(a). The central area of Fig. 8(a) is magnified and plotted in Fig. 8(b). Points (1) and (2) in Fig. 8(b) correspond to the centers of gravity of the dipole corrector magnet without and with a quadrupole magnet nearby. A shift in the magnetic center is observed. The 8.9\% reduction in $\mathrm{L}_{\text {eff }}$ results in a $15 \mathrm{~mm}$ shift for the combination of [QA, $\mathrm{StV}$ (wide)]. This reduction of the dipole corrector field and shift in the magnetic center are summarized in Table 3, for various types of dipole correctors used in the KEKB LER.
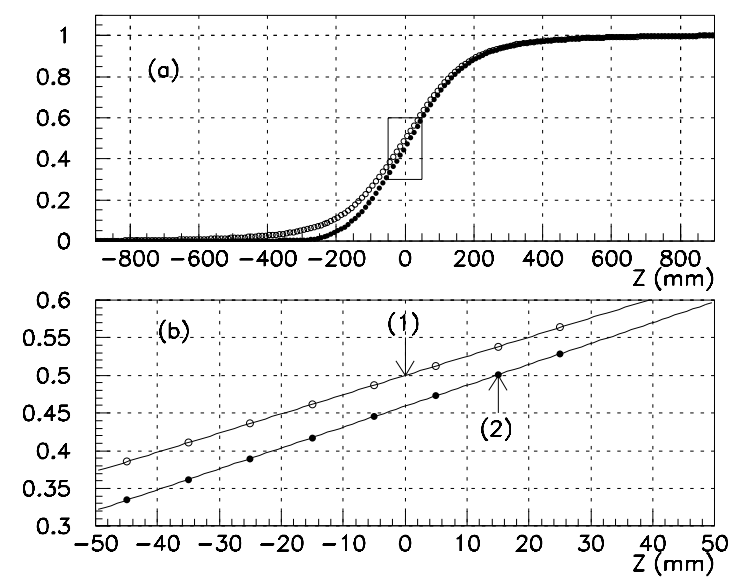

Figure 8: (a)A partial sum of the dipole field strength as a function z. (b) The difference in the magnetic centers. Point (1) and (2) are the centers of gravity of $\mathrm{StV}$ (wide) without and with a nearby quadrupole magnet. A shift is observed.

Table 3: Magnetic coupling effect on the LER dipole corrector magnets from a nearby quadrupole magnet

\begin{tabular}{|l|c|c|c|}
\hline \multicolumn{1}{|c|}{ corrector } & $\mathrm{L}_{\text {eff }}(\mathrm{mm})$ & $\Delta \mathrm{BL}$ & $\Delta$ center $(\mathrm{mm})$ \\
\hline $\mathrm{StV}(\operatorname{arc})$ & 359.00 & $6.5 \%$ & 10.3 \\
\hline $\mathrm{StH}(\operatorname{arc})$ & 319.29 & $4.2 \%$ & 3.6 \\
\hline $\mathrm{StV}($ wide $)$ & 390.27 & $8.5 \%$ & 14.7 \\
\hline $\mathrm{StH}$ (wide) & 372.88 & $8.2 \%$ & 11.6 \\
\hline
\end{tabular}

Note :The dipole field is compared at a level corresponding to $1 \mathrm{mrad}$ of kick angle. $\mathrm{L}_{\text {eff }}$ in the table is the effective length, when there is no magnetic material nearby.

\section{SUMMARY}

The magnetic coupling between quadrupole and dipole corrector magnets has been measured. Reduction in the integrated main field is observed to be $0.1 \sim 0.2 \%$ for the quadrupole magnets and 4.0 9.0\% for the dipole corrector magnets. The effects on the integrated field strength agree quantitatively with the mapping results where $\Delta \mathrm{L}_{\text {eff }}$ is measured. Magnetic coupling causes a distortion of the field distribution and thus shift in magnetic center. No significant effects on the higher multipole components are observed for the quadrupole magnets.

\section{ACKNOWLEDGMENTS}

The authors would like to thank the measurement crew for their hard work and the KEK magnet group for many useful discussions.

\section{REFERENCES}

[1] KEKB B-Factory Design Report 95-7

[2] K. Egawa and M. Masuzawa, "Preliminary Results of the KEKB Quadrupole Magnet Measurements", EPAC'98, Stockholm (1998)

[3] K. Egawa and M.Masuzawa "Field Measurements of the KEK BFactory Dipole and Wiggler Magnets", Contribution to PAC99 